\title{
Effects of Eurycoma longifolia Jack on chronic cerebral hypoperfusion-induced oxidative damage and memory deficit in rats
}

\author{
Hulol Saleh Alruhaimi ${ }^{*}$, Ahmed K. Allow², Zunariah Buyong², Muhammed Naser ${ }^{2}$, Shaikh Mizanur² \\ ${ }^{1}$ Department of Biology, Faculty of Sciences, Princess Nourah Bint Abdulrahman University, Riyadh, Kingdom of Saudi Arabia. \\ ${ }^{2}$ Department of Basic Medical Sciences, Faculty of Medicine, International Islamic University Malaysia (IIUM), Kuantan, Pahang, Malaysia.
}

\begin{tabular}{l}
\hline ARTICLE INFO \\
\hline Received on: $13 / 08 / 2018$ \\
Accepted on: 20/02/2019 \\
Available online: 18/04/2019 \\
\hline Key words: \\
Tongkat Ali, chronic cerebral \\
hypoperfusion, oxidative \\
stress, spatial learning and \\
memory, oxidative stress.
\end{tabular}

\section{INTRODUCTION}

Neurodegeneration is the progressive loss of neuronal function and structure, with increased neuronal death in Central Nervous System (CNS) (Ransohoff, 2016). Neurodegenerative diseases such as Alzheimer's disease (AD), Parkinson's disease, and many others represent a relevant health problem with age worldwide. Chronic cerebral hypoperfusion $(\mathrm{CCH})$ is associated with human aging and neurodegenerative diseases, including AD and vascular dementia (VD) (Xu et al., 2010; Yao et al., 2018). In early stage of these diseases, $\mathrm{CCH}$ is a main contributor to disease progression and memory disturbance (De la Torre, 2000). A chronic decrease in blood supply to the brain can result in an insufficient delivery of oxygen and glucose to cerebral neurons,

"Corresponding Author

Hulol Saleh Alruhaimi, Department of Biology, Faculty of Sciences, Princess Nourah bint Abdulrahman University, Riyadh, Saudi Arabia. E-mail:holo_l@hotmail.com leading to intracellular dysfunction contributing to enhanced neurodegeneration (Farkas et al., 2007). CCH induced by 2-vessel occlusion (2VO) in rats induced neuronal energy failure (Farkas et al., 2007), an increase in oxidative damage, and a reduction in antioxidant ability (Chen et al., 2016), which consequently lead to neuroinflammatory response via glial cell activation (Cechetti et al., 2012; Yao et al., 2018) and caused white matter injury (Lee et al., 2013), blood-brain barrier dysfunction (Farkas et al., 2005), free radical generation, and amyloid beta deposition (Wang et al., 2010). These factors result in cognitive decline. Therefore, alleviating oxidative stress and inflammation response could be a useful therapeutic strategy for treating $\mathrm{CCH}$-induced cognitive dysfunction.

Several studies have reported positive outcomes of varying antioxidant agents, including green tea polyphenols (Xu et al., 2010), curcumin (Gao et al., 2012), and phytochemicals such as flavonoids (Williams and Spencer, 2012), and alkaloids (Girdhar et al., 2015), in experimental models of neurodegenerative diseases. However, we selected Eurycoma longifolia (EL), most popular traditional medicinal herb in Malaysia, because 
of its anti-inflammatory and antioxidant properties. EL Jack, commonly known as Tongkat Ali, is a flowering plant of the family Simaroubaceae; it is used in South-East Asian countries (Rehman et al., 2016). The wide range of pharmacological effects of EL is associated with its different bioactive compounds, including quassinoids, canthin-6-ones, triterpenes, and $\beta$-carboline alkaloids (Hajjouli et al., 2014), as well as flavonoids and phenolic compounds (Khanam et al., 2015). The important pharmacological properties of EL have been reported to be its antidiabetic, antitumor, antimalarial, anticancer, anti-inflammatory, and antioxidant activities (Hai Dang et al., 2016; Rashid et al., 2009; Varghese et al., 2013); however, its neuropharmacological properties have not been examined to date. Therefore, the present study aimed to evaluate the potential effect of EL extract in $\mathrm{CCH}$ -induced spatial learning and memory impairment and oxidative damage in rat.

\section{MATERIALS AND METHODS}

\section{Methanolic extraction of Eurycoma longifolia}

Powdered EL roots were purchased from Delima Jelita Herbs Pvt. Ltd. (Alor Setar, Kedah, Malaysia). The commercialized plant materials were forest produce of Sik district, Kedah, Malaysia, as indicated on the package. The obtained root powder was macerated with $70 \%$ methanol using a Soxhlet extractor for 8 hours. Subsequently, the extract was filtered and evaporated using a rotary evaporator (Buchi). The final yield of the prepared extracts was $22.4 \mathrm{~g}$, and it was stored at $-4^{\circ} \mathrm{C}$ for further study (Khanam et al., 2015).

\section{Animals}

After approval from Institutional Animal Ethics Committee of IACUC-IIUM [NO. (2016/11)(70)], 33 male Sprague-Dawley rats (age: approximately 7 months, weight: 250 $380 \mathrm{~g}$ at $2 \mathrm{VO}$ surgery) were used in this study. Rats were housed (pairs/cage) at a temperature of $25^{\circ} \mathrm{C} \pm 1{ }^{\circ} \mathrm{C}$ and relative humidity of $45 \%-59 \%$, with 12 -hours dark: light cycle and food pellets and tap water ad libitum. The study protocols were in accordance with the guidelines of the National Institute of Health with respect to the care and use of laboratory animals.

\section{Experimental design}

After a 1-week acclimatization period, the rats were randomly divided into the following experimental groups, each group including 11 rats: sham group; untreated 2VO group; and $\mathrm{EL}+2 \mathrm{VO}$ group, $2 \mathrm{VO}$ rats treated with EL extract $(100 \mathrm{mg} / \mathrm{kg} /$ day). The EL extract was administered by oral gavage for 3 days before the date of $2 \mathrm{VO}$ surgery and continued later until the end of postoperative week 8 (Fig. 1).

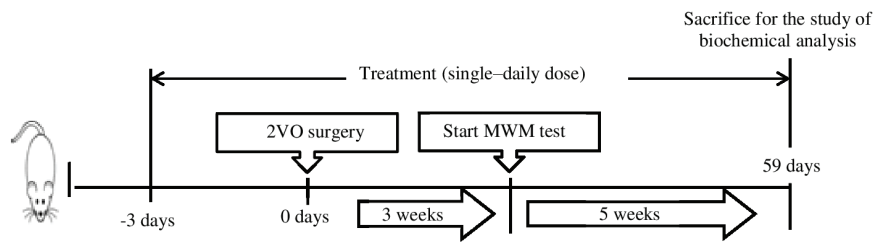

Figure 1. Flowchart of the experimental design.

\section{VO surgery}

$\mathrm{CCH}$ was induced in the rats as previously published (Azzubaidi et al., 2012). The rats received a combined intraperitoneal injection of ketamine $(90 \mathrm{mg} / \mathrm{kg})$ and xylazine $(20$ $\mathrm{mg} / \mathrm{kg}$ ) as anesthetic agents. A small midline incision (2-cm long) was made in the neck skin; thereafter, the connective tissue and muscles were separated to identify the common carotid arteries, which were subsequently doubly ligated with 3-0 silk sutures and cut between the ligations. The sham group underwent the same procedure but without such a ligation.

\section{Morris water maze test}

Cognitive function of the rats was evaluated using the Morris water maze (MWM) task, on days 21-25 after 2VO injury. The maze involved a black circular tank (diameter, $200 \mathrm{~cm}$; height, $60 \mathrm{~cm})$ filled with water $\left(24^{\circ} \mathrm{C} \pm 26^{\circ} \mathrm{C}\right)$ to a depth of $40 \mathrm{~cm}$. A hidden circular black platform (diameter, $10 \mathrm{~cm}$ ) was submerged $2 \mathrm{~cm}$ below the water surface and placed at the same location in the southwest quadrant throughout the training period. In addition, colored posters were pasted on the tank wall to aid the rats in learning the platform location. The water pool was divided by two hypothetical lines into four imaginary quadrant zones (North, South, East, and West) of an equal surface area, representing four starting points for the test. All rats were subjected to four trials per day for four consecutive days. On day 21, each rat was lowered into the pool facing the wall and allowed 120 seconds to find the platform. If the rat failed to find the platform within this time period, it was gently guided to it and allowed a 30 -second rest on the platform before being taken out from the maze. The constant daily sequence of starting points for all test trials was randomly selected until the completion of four starting points per rat for each day and the sequence was not repeated on the next test. The swimming distance and escape latency until reaching the hidden platform were recorded using the Ethovision video tracking system (Noldus, The Netherlands). On day 5, each rat was subjected to a single 60 -second trial without the platform, and the crossing times to the platform zone and the time spent in the target quadrant were recorded (Azzubaidi et al., 2012).

\section{Biochemical analyses}

After 8 weeks, the animals were anesthetized with diethylether, and blood samples were collected via the retroorbital plexus to obtain serum, which was separated by centrifuging the blood at 3,000 g for 15 minutes at $4^{\circ} \mathrm{C}$ and then stored at $-80^{\circ} \mathrm{C}$ until biochemical analyses. The malondialdehyde (MDA) concentration, superoxide dismutase (SOD) activity, and glutathione (GSH) levels were measured using a biochemistry assay kit (BioVision Inc., San Francisco, CA). Moreover, the C-reactive protein (CRP) concentration was measured using an enzyme-linked immunosorbent assay kit (Cloud-Clone Corp Systems, Houston, TX), according to the manufacturers' instructions.

\section{Statistical analyses}

All results are presented as the mean \pm standard error of mean. The MWM data were analyzed using repeated-measures analysis of variance (ANOVA), followed by post-hoc comparison 
to assess the significance of difference among the experimental groups. The other data were analyzed using one-way ANOVA. If the overall $p$-value was statistically significant $(p<0.05)$, the groups were further compared using the post hoc least-significant difference test.

\section{RESULTS}

\section{Effects of Eurycoma longifolia extracts on spatial learning and} memory

Three weeks after the $2 \mathrm{VO}$ surgery, the rats were subjected to 4 days of trials in the MWM to determine their spatial learning ability. As shown in Fig. 2A, the swimming distance to reach the platform in sham group $(49.21 \pm 27.44 \mathrm{~cm})$ and $\mathrm{EL}+2 \mathrm{VO}$ treated rats $(62.29 \pm 40.29 \mathrm{~cm})$ was significantly shorter within 4 days of the acquisition phase compared to $2 \mathrm{VO}$ group $(71.20$ $\pm 49.45 \mathrm{~cm})(p<0.001, p<0.05$, respectively). Moreover, the swimming distance was significantly longer in $\mathrm{EL}+2 \mathrm{VO}$ group $(p$ $<0.001$ ) throughout 4 days of acquisition phase when compared to sham group (Fig. 2A). In addition, the results of the present study showed that the untreated $2 \mathrm{VO}$ group $(100 \pm 37.81$ seconds $)$ and $\mathrm{EL}+2 \mathrm{VO}$ group $(82.72 \pm 25.27$ seconds) had significantly $(p<$ $0.001, p<0.05$, respectively) higher escape latencies to find the platform within 4 days of acquisition phase compared to the sham group (51.09 \pm 13.18 seconds), as shown in Fig. 2B. In contrast, the $\mathrm{EL}+2 \mathrm{VO}$ treated groups required significantly $(p<0.05$, respectively) less time to find the platform during training phase when compared with untreated $2 \mathrm{VO}$ group (Fig. 2B). In the probe trial (Fig . 3), the untreated $2 \mathrm{VO}$ rats spent less time in the target zone $(p<0.01)$ than did the sham rats, while the time spent by the $\mathrm{EL}+2 \mathrm{VO}$ groups in the target zone significantly increased $(p<$ 0.05 ) compared with those in the untreated $2 \mathrm{VO}$ group.

\section{Effects of Eurycoma longifolia extracts on MDA concentration, GSH levels, and SOD activity in $\mathrm{CCH}$ rats}

The MDA concentration was significantly higher $(p<$ 0.001 ) and the GSH levels and SOD activity were lower (all $p$ $<0.001$; Table 1) in the untreated $2 \mathrm{VO}$ group than in the sham group. In contrast, the oral administration of EL (100 mg/ $\mathrm{kg}$ /day) reduced the MDA concentration $(p<0.001)$ and significantly enhanced GSH levels and SOD activity (all $p<0.001$ ) in 2VO rats compared with untreated $2 \mathrm{VO}$ rats.

\section{Effects of Eurycoma longifolia extracts on CRP concentration in $\mathrm{CCH}$ rats}

The CRP concentration was significantly increased in the untreated 2VO group ( $p<0.001$; Table 1) compared with the sham group, whereas the CRP concentration was significantly decreased in the EL $+2 \mathrm{VO}$ groups than in the untreated $2 \mathrm{VO}$ group $(p<$ $0.001, p<0.05$, respectively).

\section{DISCUSSION}

$\mathrm{CCH}$ induced by permanent bilateral occlusion of the common carotid arteries $(2 \mathrm{VO})$ in rats results in a chronic decrease in the cerebral blood flow, which is considered to induce hippocampal neurodegeneration and its resultant loss of memory. The 2VO model is considered acceptable for evaluating the pathophysiology of cognitive failure related to cerebral circulation
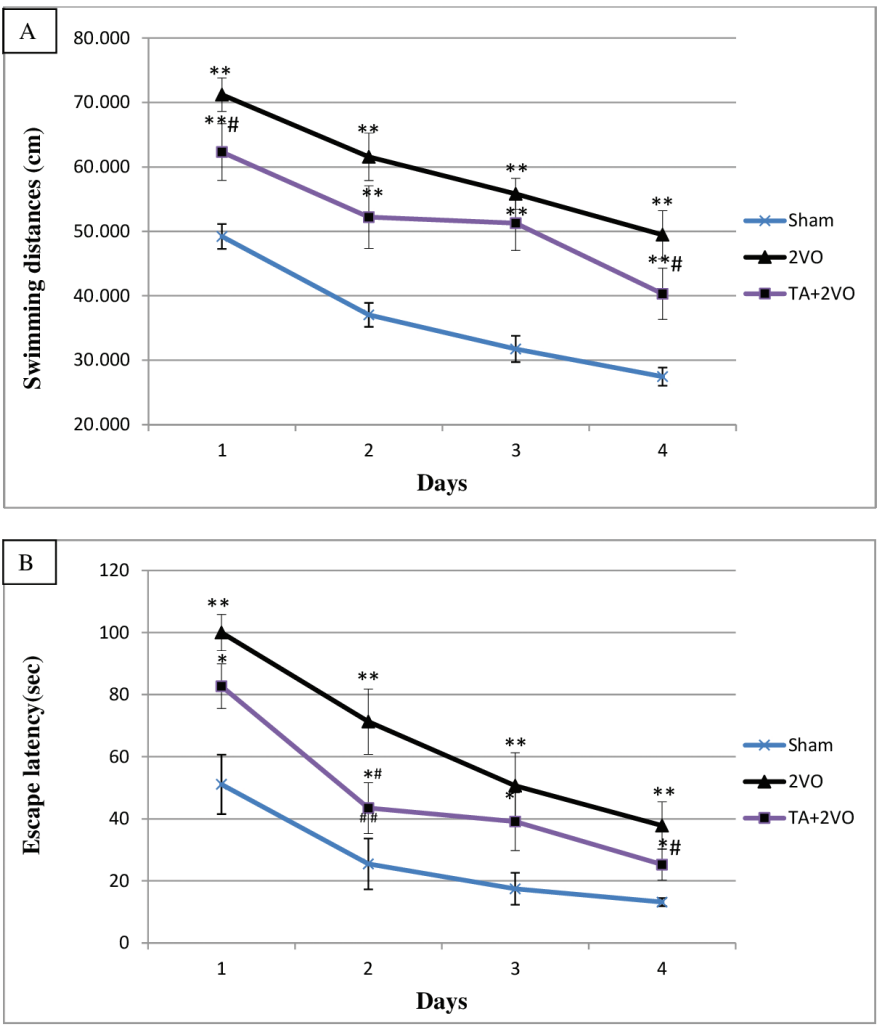

Figure 2. MWM test shows intergroup differences in swimming distance (A) and escape latency (B). ${ }^{* \#} p<0.05,{ }^{* * \#} p<0.01$. ${ }^{*}$ Compared with the sham group. ${ }^{\sharp}$ Compared with the untreated $2 \mathrm{VO}$ group.

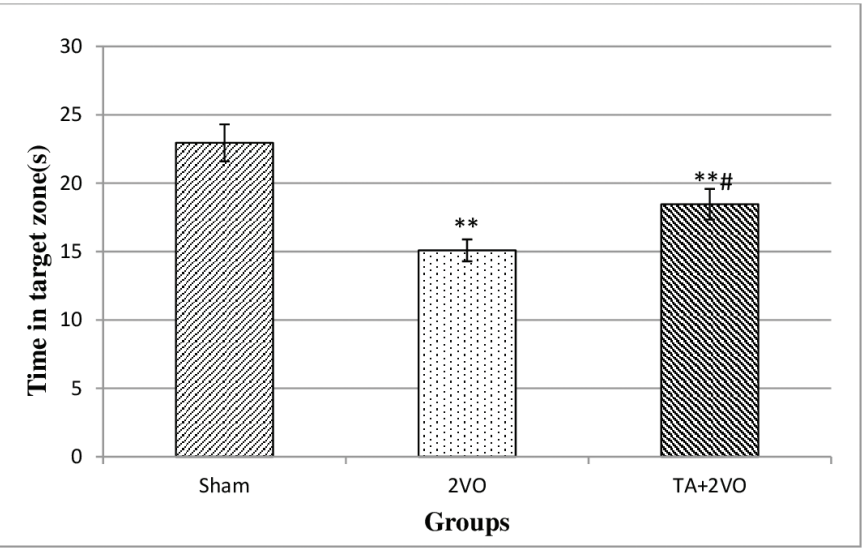

Figure 3. Spent time in the target quadrant(s) in the probe trial of reference memory in experimental groups.

disturbance, as well as to investigate the potential therapeutic effects of various neuroprotective agents (Farkas et al., 2007). In recent years, significant preclinical studies have been conducted with respect to traditional medicinal herbs as neuroprotective agents; these studies revealed positive results through the attenuation of oxidative stress and neuroinflammation response that affect cerebral neurons, regardless of the cause of brain insult. Therefore, we investigated the antioxidant and neuroprotective effects of EL in a rat model of $\mathrm{CCH}$-induced neurodegeneration. 
Table 1. Effect of EL extract on serum MDA, GSH, SOD, and CRP levels in rats.

\begin{tabular}{lcccc}
\hline Group & MDA $(\mathbf{n m o l} / \mathbf{m l})$ & GSH $(\mathbf{n g} / \mathbf{m l})$ & SOD $(\mathbf{U} / \mathbf{m l})$ & CRP $(\mathbf{n g} / \mathbf{m l})$ \\
\hline Sham & $0.333 \pm 0.007$ & $29.45 \pm 0.652$ & $29.80 \pm 1.50$ & $1.65 \pm 0.157$ \\
2 VO & $0.492 \pm 0.034^{* *}$ & $15.82 \pm 1.292^{* *}$ & $14.50 \pm 1.683^{* *}$ & $3.30 \pm 0.266^{* *}$ \\
EL +2 VO & $0.407 \pm 0.008^{* * * *}$ & $22.58 \pm 1.238^{* * * \#}$ & $24.35 \pm 1.147^{* \# *}$ & $2.54 \pm 0.25^{* * *}$ \\
\hline
\end{tabular}

Data are shown as the mean \pm standard error of the mean. *\#p<0.05,**\#\#p<0.01. "Compared with the sham group. ${ }^{\#}$ Compared with the untreated $2 \mathrm{VO}$ group ( $n=11$ for each group).

Sham: sham-operated group, EL + 2VO: Rats had 2VO treated with EL roots extract.

In the present study, the MWM test revealed that spatial learning and memory were significantly impaired in the untreated $2 \mathrm{VO}$ rats compared to the sham rats, indicating an association between $\mathrm{CCH}$ and cognitive deficit. This finding is consistent with those of previous reports (Azzubaidi et al., 2012; Damodaran et al., 2014; Zhang et al., 2010), suggesting that the memory impairment after $\mathrm{CCH}$ results from neuronal damage and oxidative stress response in the hippocampus. This gradual impairment in memory function increased with the duration of ischemia (Damodaran et al., 2014; Liu et al., 2005). The chronic administration of EL at a dose of $100 \mathrm{mg} / \mathrm{kg}$ /day showed a slight improvement in cognitive function compared with untreated $2 \mathrm{VO}$ rats although both the escape latency time and swimming distance were extremely longer than those in the sham group; however, the EL treated rats spent longer time in the target quadrant Southwest (SW) as compared to the $2 \mathrm{VO}$ group. A possible explanation for this result might be that the dosage of EL extract was not enough to attenuate subcellular alterations and transmission failure caused by $\mathrm{CCH}$ in rats. Nevertheless, there has been no animal and clinical research focusing on the effect of EL on neurodegenerative diseases.

Previous studies have reported that free radicals can induce neuronal degeneration and their probable involvement in the pathogenesis of neuronal damage in neurodegenerative disorders, including AD and VD (Chong et al., 2005; Markesbery, 1997). Our study also showed that lipid peroxidation was markedly increased, as indicated by the increased MDA concentration and decreased GSH levels and SOD activity in the untreated $2 \mathrm{VO}$ rats. This finding suggested that $\mathrm{CCH}$ induced the production of free radicals that could destroy the antioxidant defense system of the brain, resulting in their decreased levels. These results are in concordance with the theory of oxidative injury, in which $2 \mathrm{VO}$ could induce oxidative stress and lead to cellular damage (Cechetti et al., 2012; Mirzaie et al., 2016; Yao et al., 2018). GSH and SOD are the most abundant brain antioxidants and play an important role in protecting the brain from oxidative injury (Cechetti et al., 2012). The decrease in GSH levels might be caused by the decreased synthesis of GSH, which is highly sensitive to ischemic disturbance at the cellular level (Xie et al., 1989), while the decreased SOD activity in ischemic rats may be caused by an increased consumption of superoxide radicals as a result of increased oxidative stress (Tanaka et al., 2002). The EL extract significantly decreased MDA levels and increased the antioxidant ability in $2 \mathrm{VO}$ rats. A possible explanation for this is that the presence of various bioactive ingredients, including phenolic compound, alkaloids, flavonoids, and glycosides, in the root extract of E. longifolia (Khanam et al., 2015) confers some degree of neuroprotection by diminishing oxidative stress caused by free radicals. Varghese et al. (2013) reported that the EL root extract showed significant antioxidant activity by free radical scavenging in vitro. Based on these results, the antioxidant activity of EL may underlie a modest improvement in cognitive functions. However, there exists a gap in research on the antioxidant activity of EL; thus, we recommend conducting more studies on the antioxidant mechanisms of EL. Another important finding is that the levels of CRP, an acute-phase protein, were increased after $\mathrm{CCH}$. This result may be explained by the increase in oxidative stress that caused glia cell activation, which consequently leads to increased levels of proinflammatory cytokines, including interleukin (IL)-1 $\beta$ and IL-6 (Cechetti et al., 2012), which are known to alter CRP expression (Juma et al., 2011). CRP can stimulate phagocytosis and activate the classical complement pathway, which contributes to the increasing level of inflammatory responses, and thus, is implicated in neurodegeneration (Bonifati and Kishore, 2007). This result is in agreement with those reported by Juma et al. (2011) who reported that the CRP level was greater in $\mathrm{CCH}$ rats and reached statistical significance at day 3 following $2 \mathrm{VO}$ injury. This finding corroborates the observation of activated microglia from day 3 onward after 2VO surgery (Juma et al., 2011; Wang et al., 2010; Watanabe et al., 2006). Daily treatment with EL alleviated the increasing CRP concentration after $\mathrm{CCH}$, suggesting that the active constituents in EL extract have a protective effect on $\mathrm{CCH}$ by reducing the inflammatory response. These data are in accordance with those of previous studies, which reported the anti-inflammatory effects of EL root extract (Hai Dang et al., 2016; Varghese et al., 2013). The anti-inflammatory effects of EL might reflect its content of alkaloid and quassinoid compounds, which can inhibit the transcriptional factor nuclear factor- $\kappa \mathrm{B}$ (Tran et al., 2014), thus reducing pro-inflammatory factors such as Cyclooxygenase-2 (COX-2) and Inducible nitric oxide (iNO) (Hoesel and Schmid, 2013). Furthermore, the EL extract increased the anti-inflammatory activity in a concentration-dependent manner (Varghese et al., 2013). Overall, oral treatment with EL extract at a dose of $100 \mathrm{mg} / \mathrm{kg} /$ day exhibited a modest prevention effect on memory and learning, oxidative stress, and neuroinflammation in $\mathrm{CCH}$ model; it can be assumed that an antioxidant and anti-inflammatory effect were the mechanism behind this mnemonic action.

\section{CONCLUSION}

To the best of our knowledge, this study is the first to investigate the neuroprotective effects of $\mathrm{EL}$ in $\mathrm{CCH}$ induced by $2 \mathrm{VO}$ surgery. These findings revealed that EL may have a potential neuroprotective effect on $\mathrm{CCH}$ by enhance antioxidant ability and reduce lipid peroxidation and inflammation, which may underlie cognitive function improvement. However, this is a preliminary study; which has methodological limitations, thus, further studies should focus on the neuroprotective mechanisms of EL extract. For instance, it will be fruitful in future studies if we can measure the anti-inflammatory effect of EL by using immunohistochemistry analysis in a different area of the brain, including the hippocampus and cortex. In addition, we recommend to conduct more studies to identify the active compounds in EL responsible for neuroprotective effects. 


\section{ACKNOWLEDGMENTS}

This study was supported by Princess Nourah bint Abdulrahman University, Riyadh, Kingdom of Saudi Arabia.

\section{CONFLICT OF INTEREST}

There are no conflicts of interest.

\section{REFERENCES}

Azzubaidi MS, Saxena AK, Talib NA, Ahmed QU, Dogarai BB. Protective effect of treatment with black cumin oil on spatial cognitive functions of rats that suffered global cerebrovascular hypoperfusion. Acta Neurobiol Exp, 2012; 72:154-65.

Bonifati DM, Kishore U. Role of complement in neurodegeneration and neuroinflammation. Mol Immunol, 2007; 44:9991010 .

Cechetti F, Worm PV, Elsner VR, Bertoldi K, Sanches E, Ben J, Siqueira IR, Netto CA. Forced treadmill exercise prevents oxidative stress and memory deficits following chronic cerebral hypoperfusion in the rat Neurobiol Learn Mem, 2012; 97:90-6.

Chen WW, Zhang X, Huang WJ. Role of neuroinflammation in neurodegenerative diseases. Mol Med Rep, 2016; 13:3391-6.

Chong ZZ, Li F, Maiese K. Oxidative stress in the brain: novel cellular targets that govern survival during neurodegenerative disease. Prog Neurobiol, 2005; 75:207-46.

Damodaran T, Hassan Z, Navaratnam V, Muzaimi M, Ng G, Müller CP, Dringenberg HC. Time course of motor and cognitive functions after chronic cerebral ischemia in rats. Behav Brain Res, 2014; 275:252-8.

De la Torre JC. Critically attained threshold of cerebral hypoperfusion: can it cause Alzheimer's disease? Ann NY Acad Sci, 2000; 903:424-36.

Farkas E, Annaházi A, Institóris Á, Mihály A, Luiten PG, Bari F. Diazoxide and dimethyl sulphoxide alleviate experimental cerebral hypoperfusion-induced white matter injury in the rat brain. Neurosci Lett, 2005; 373:195-9.

Farkas E, Luiten PGM, Bari F. Permanent, bilateral common carotid artery occlusion in the rat: a model for chronic cerebral hypoperfusion-related neurodegenerative diseases. Brain Res Rev, 2007; $54: 162-80$.

Gao M, Liu L, Zhang P, Yu G, Li Y. Neuroprotective effect of curcumin involved in increasing the protein levels of UCP2 and inhibiting oxidative stress induced by chronic cerebral ischemia in vitro. Mol Neurodegener, 2012; 7:S26.

Girdhar S, Girdhar A, Verma SK, Lather V, Pandita D. Plant derived alkaloids in major neurodegenerative diseases: from animal models to clinical trials. J Ayurvedic Herb Med, 2015; 1:91-100.

Hai Dang N, Choo YY, Tien Dat N, Hoai Nam N, Van Minh C, Lee JH. 7-Methoxy-(9H- $\beta$-carbolin-1-il)-(E)-1-propenoic acid, a $\beta$-carboline alkaloid from Eurycoma longifolia, exhibits anti-inflammatory effects by activating the Nrf2/heme oxygenase-1 pathway. J Cell Biochem, 2016; 117:659-70.

Haijouli S, Chateauvieux S, Teiten MH, Orlikova B, Schumacher M, Dicato M, Diederich M. Eurycomanone and eurycomanol from Eurycoma longifolia Jack as regulators of signaling pathways involved in proliferation, cell death and inflammation. Molecules, 2014; 19:14649-66.

Hoesel B, Schmid JA. The complexity of NF- $\mathrm{kB}$ signaling in inflammation and cancer. Mol Cancer, 2013; 12:86.

Juma WM, Lira A, Marzuk A, Marzuk Z, Hakim AM, Thompson CS. C-reactive protein expression in a rodent model of chronic cerebral hypoperfusion. Brain Res, 2011; 1414:85-93.

Khanam Z, Wen CS, Bhat IUH. Phytochemical screening and antimicrobial activity of root and stem extracts of wild Eurycoma longifolia Jack (Tongkat Ali). J King Saud Univ Sci, 2015; 27:23-30.

Lee KM, Bang JH, Han JS, Kim BY, Lee IS, Kang HW, Jeon WK. Cardiotonic pill attenuates white matter and hippocampal damage via inhibiting microglial activation and downregulating ERK and p38 MAPK signaling in chronic cerebral hypoperfused rat. BMC Compl Altern Med, $2013 ; 13: 334$

Liu HX, Zhang JJ, Zheng P, Zhang Y. Altered expression of MAP-2, GAP-43, and synaptophysin in the hippocampus of rats with chronic cerebral hypoperfusion correlates with cognitive impairment. Mol Brain Res, 2005; 139:169-77.

Markesbery WR. Oxidative stress hypothesis in Alzheimer's disease. Free Radic Biol Med, 1997; 23:134-47.

Mirzaie M, Khalili M, Kiasalari Z, Roghani M. Neuroprotective and antiapoptotic potential of trigonelline in a striatal 6-hydroxydopamine rat model of Parkinson's disease. Neurophysiology, 2016; 48:176-83.

Ransohoff RM. How neuroinflammation contributes to neurodegeneration. Science, 2016; 353:777-83.

Rashid M, Kumar S, Ahmad B. Medical uses of Eurycoma longifolia jack: a review. Pharm Res, 2009; 2:70-8.

Rehman SU, Choe K, Yoo HH. Review on a traditional herbal medicine, Eurycoma longifolia jack (Tongkat Ali): its traditional uses, chemistry, evidence-based pharmacology and toxicology. Molecules, 2016; $21: 331$.

Tanaka Y, Tran PO, Harmon J, Robertson RP. A role for glutathione peroxidase in protecting pancreatic $\beta$ cells against oxidative stress in a model of glucose toxicity. Proc Nat Acad Sci, 2002; 99:12363-8.

Tran TVA, Malainer C, Schwaiger S, Atanasov AG, Heiss EH,

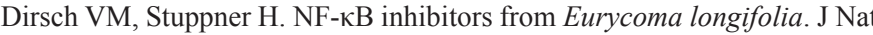
Prod, 2014; 77:483-8.

Varghese CP, Ambrose C, Jin SC, Lim YJ, Keisaban T. Antioxidant and anti-inflammatory activity of Eurycoma longifolia jack, a traditional medicinal plant in Malaysia. Int J Pharm Sci Nanotech, 2013; $5: 1875-8$

Wang X, Xing A, Xu C, Cai Q, Liu H, Li L. Cerebrovascular hypoperfusion induces spatial memory impairment, synaptic changes, and amyloid- $\beta$ oligomerization in rats. J Alzheimer's Dis, 2010; 21:813-2.

Watanabe T, Zhang N, Liu M, Tanaka R, Mizuno Y, Urabe T. Cilostazol protects against brain white matter damage and cognitive impairment in a rat model of chronic cerebral hypoperfusion. Stroke, 2006; 37:1539-45.

Williams RJ, Spencer JP. Flavonoids, cognition, and dementia: actions, mechanisms, and potential therapeutic utility for Alzheimer disease. Free Rad Biol Med, 2012; 52:35-45.

Xie Y, Mies G, Hossmann KA. Ischemic threshold of brain protein synthesis after unilateral carotid artery occlusion in gerbils. Stroke, $1989 ; 20: 620-6$.

Xu Y, Zhang JJ, Xiong L, Zhang L, Sun D, Liu H. Green tea polyphenols inhibit cognitive impairment induced by chronic cerebral hypoperfusion via modulating oxidative stress. J Nutr Biochem, 2010; 21:741-8

Yao ZH, Yao XL, Zhang Y, Zhang SF, Hu JC. Luteolin could improve cognitive dysfunction by inhibiting neuroinflammation. Neurochem Res, 2018; 43:806-20.

Zhang L, Fu F, Zhang X, Zhu M, Wang T, Fan H. Escin attenuates cognitive deficits and hippocampal injury after transient global cerebral ischemia in mice via regulating certain inflammatory genes. Neurochem Int, 2010; 57:119-27.

How to cite this article:

Alruhaimi HS, Allow AK, Buyong Z, Naser M, Mizanur S. Effects of Eurycoma longifolia Jack on chronic cerebral hypoperfusion-induced oxidative damage and memory deficit in rats. J Appl Pharm Sci, 2019; 9(04):077-081. 